\title{
Microfluidic devices manufacturing combining stereolithography and pulsed laser ablation
}

\author{
Bastián Carnero ${ }^{l}$, Carmen Bao-Varela ${ }^{l}$, Ana Isabel Gómez-Varela ${ }^{l}$, and María Teresa Flores-Arias ${ }^{1, *}$ \\ ${ }^{1}$ Photonics4Life research group, Department of Applied Physics, Facultade de Física and Facultade de Óptica e Optometría, Universidade \\ de Santiago de Compostela, Campus Vida, E-15782 Santiago de Compostela, Spain
}

\begin{abstract}
D printing has revolutionized the field of microfluidics manufacturing by simplifying the typical processes offering a considerable accuracy and user-friendly procedures. For its part, laser ablation proves to be a versatile technology to perform detailed surface micropatterning. A hybrid technique that combines both technologies is proposed, employing them in their most suitable range of dimensions. This technique allows to manufacture accurate microfluidics devices as the one proposed: a microchannel, obtained using a stereolithographic printer, coupled with an array of microlenses, obtained by pulsed laser ablation of a 3D printed master.
\end{abstract}

\section{Introduction}

Optical technologies have proven their versatility in the manufacturing of microfluidic devices of different sizes, geometries, and functionalities. Historically, photolithography or reactive ion etching have been the most used manufacturing techniques in microfluidics [1], requiring the manipulation of very complex masks and producing a considerably polluting chemical waste. These techniques, although precise, show limitations by only allowing superficial structuring of various photoresists and polymers. This has recently changed thanks to advances in lasers, promoting new technologies to appear. In this regard, stereolithographic 3D printers stand out by allowing to structure many materials in volume with high structural complexity [2], that are increasingly used for manufacturing microfluidic devices for biological applications [3]. Laser ablation has also simplified the micropatterning of a vast quantity of materials since it is a self-sufficient process that does not need of posterior chemical treatments neither masks to pattern the surfaces. Recent works have even proposed strategies to perform laser ablation of 3D surfaces in real time [4].

This contribution presents a novel hybrid technique for the manufacturing of microfluidic devices that combines laser ablation with an emerging 3D printing optical technology: stereolithography (SLA). In this technique, each technology is used to manufacture structures in the dimension range that is most suitable for it. Thereby, an SLA 3D printer is used to obtain 3D printed masters in resin with microchannels of hundreds of microns. Meanwhile, laser ablation is performed on the surface of this printing resin to create fine detailed structures in the range of tens of microns, unreachable for most current 3D printers. Finally, the channels and micropatterned structures of the masters are replicated by soft lithography of polydimethylsiloxane (PDMS). The latter one of the most used polymers to manufacture biological microfluidic devices [5], given its optical transparency to visible and UV light, permeability to gases, elasticity, and biocompatibility.

\section{Materials and Methods}

A Form 3B SLA printer (Formlabs, Somerville, Massachusetts) was used in this work to manufacture the microchannel master as well as the substrate for ablation. SLA printers have a tank in which a liquid printing resin is deposited so a proper light source (generally a laser) performs a selectively layer-by-layer photopolymerization (Fig. 1).

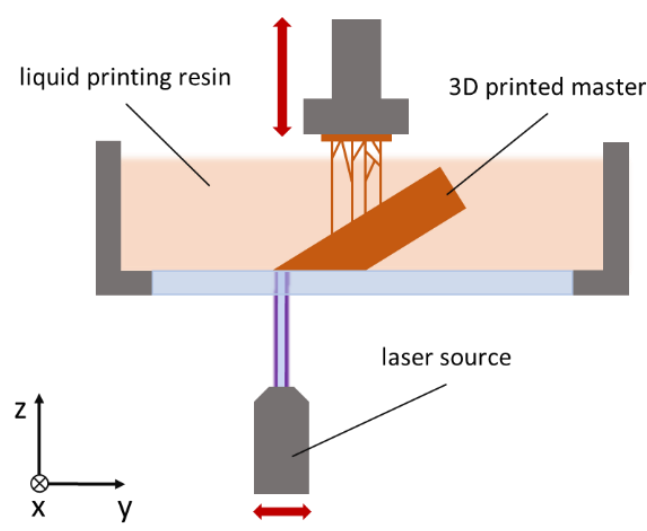

Fig. 1. SLA 3D printing principle.

The used Form 3B printer employs Low Force Stereolithography (LFS), a novel technique that improves the precision and accuracy of printed pieces, by reducing the manufacturing stresses that the pieces suffer during

\footnotetext{
* Corresponding author: maite.flores@usc.es
} 
the printing. For this, a resin tank with a flexible sheet at its base that can be gradually deformed when the piece is pressed against it is used. For the polymerization of the resin, the laser is always addressed perpendicular to the piece thanks to a galvanometric system. Commercial Model V2 resin from Formlabs was selected as printing resin given the high precision it offers, its good performance when replicating polymers and the good response offered to laser ablation. In the postcuring process, the pieces are washed in isopropanol and introduced into a UV chamber for 60 minutes at $60^{\circ} \mathrm{C}$.

Pulsed laser ablation (PLA) of the 3D printed substrate was performed in the STELA laser of the Laser Laboratory for Acceleration and Applications (L2A2), a research facility of the University of Santiago de Compostela, using a beam of $800 \mathrm{~nm}$ of wavelength, pulse duration of $35 \mathrm{fs}$ and repetition rate of $1 \mathrm{kHz}$. The laser was focused on the surface of the resin employing a Mitutoyo M Plan APO NIR 20x objective.

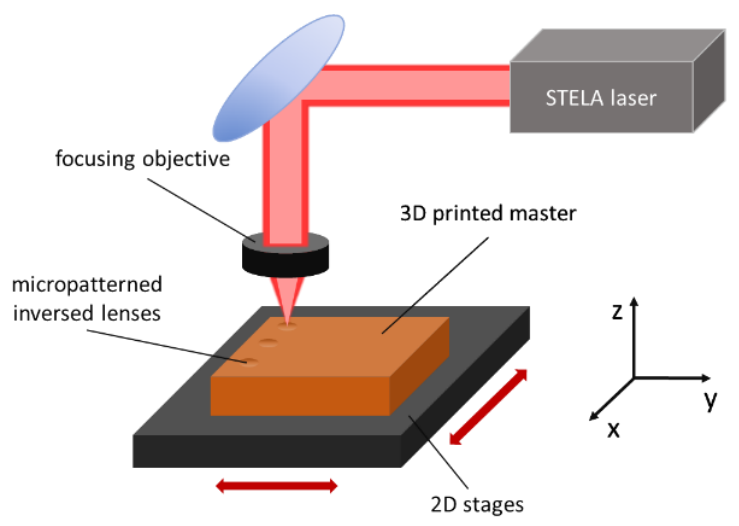

Fig. 2. PLA micropatterning principle.

PDMS was selected as soft lithography replication polymer given the previous introduced reasons. The PDMS was prepared from Sylgard 184 elastomer by mixing the monomer and the curing agent in a ratio 10:1. The mixture was then deposited over the structures fabricated by 3D printing and ablation (with walls added to avoid losses of liquid polymer) forming a thin layer, introduced in a vacuum chamber for $40 \mathrm{~min}$ at $400 \mathrm{mbar}$ to remove bubbles produced during the mixing process and cured in an oven for $12 \mathrm{~h}$ at $60^{\circ} \mathrm{C}$.

For bonding, a Diener Zepto (Diener, Ebhausen, Germany) plasma cleaner was employed, exposing the PDMS surfaces to an oxygen plasma. First, vacuum was performed in the chamber until reach 0.1 mbar. Next, oxygen was introduced into the chamber during 7 minutes until a pressure of 0.4 mbar was reached. Then, the generator was activated, and the plasma initialized. Power was fixed at $50 \mathrm{~W}$ and exposure lasted for 20 seconds, for glass-PDMS bonding, and 10 seconds, for PDMS-PDMS bonding. Next, the plasma cleaner vented and reached ambient pressure. Finally, the pieces were put in contact and introduced into a furnace to receive a thermal treatment (to promote full bonding) of 20 minutes at 100 ${ }^{\circ} \mathrm{C}$.

\section{Results and Discussion}

To test the possibilities offered by the proposed hybrid technique, a sandwiched microfluidic device was manufactured by bonding two structured layers of PDMS to a microscope slide. The top layer (cover) was obtained from a master superficially micropatterned by PLA, featuring an array of microlenses for focusing light on the microchannel presented in the bottom layer (substrate). The proposed device could be used to perform selective photopolymerization of a small photocurable sample that flows through the microchannel [6].

\subsection{Cover manufacturing: microlenses arrays}

For the manufacture of the cover, a wafer was printed in Model resin, on which surface ablation tests were performed, as shown in Fig. 2. By PLA, it was possible to create inverse microlenses in the resin, to be later replicated with PDMS and obtain various arrays of microlenses with different geometries and periods (Fig. $3)$.
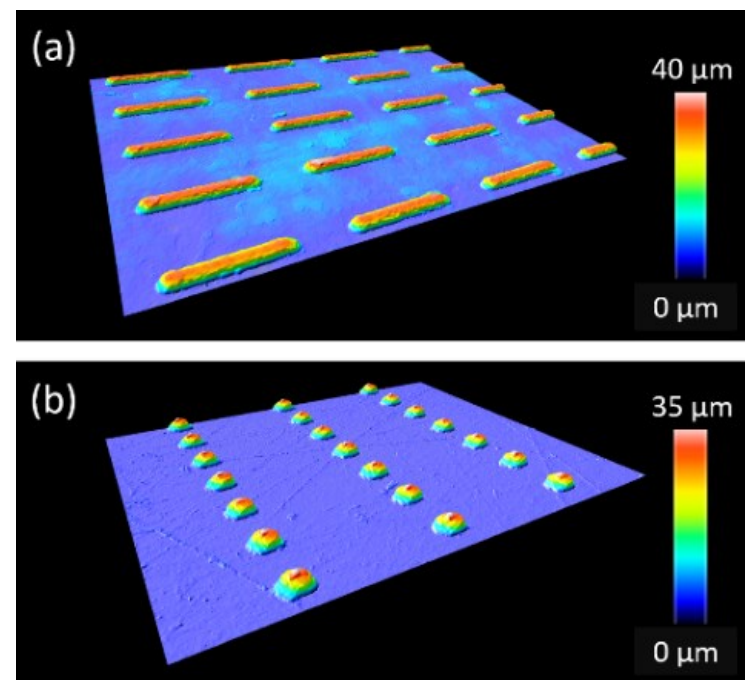

Fig. 3. Confocal images of a) cylindrical and b) hemispherical microlenses of PDMS.

Thereby, cylindrical lenses of $35 \mu \mathrm{m}$ of radius, approximately $125 \mu \mathrm{m}$ of focal distance and $150 \mu \mathrm{m}$ of length were patterned with $20 \mathrm{~mm} / \mathrm{s}$ of velocity and 30 $\mathrm{mW}$ of power on the unpolished resin surface, forming the array showed in Fig. 3a, with $240 \mu \mathrm{m}$ of period. Moreover, hemispherical lenses of $25 \mu \mathrm{m}$ of radius and approximately $90 \mu \mathrm{m}$ of focal distance were patterned with $60 \mathrm{~mW}$ of power on the polished surface, forming the array showed in Fig. 3b, with $240 \mu \mathrm{m}$ of period.

The different geometries of the microlenses achievable with this hybrid technique will determinethe way in which the liquid sample can be selective photopolymerized, allowing to obtain a wide range of polymerized microdroplets, with the desired size and sphericity for each specific application. 


\subsection{Substrate manufacturing: microchannel}

The second manufactured element was a master to obtain a square microchannel of $200 \mu \mathrm{m}$ in height. The outward microchannel with connectors was designed on the bottom of a 20x40 mm box, using a CAD-like software (Fig. 4a). The resin printed piece was postprocessed to optimize its mechanical properties and then easily replicated by PDMS soft lithography, forming the inward replica of the previous structure. A $300 \times 500 \mu \mathrm{m}$ array of hemispherical microlenses was fabricated in PDMS and bonded over the start of the channel (Fig 4b). Finally, the layers were bonded to a microscope slide, to obtain a fully functional and easily handled microfluidic device.
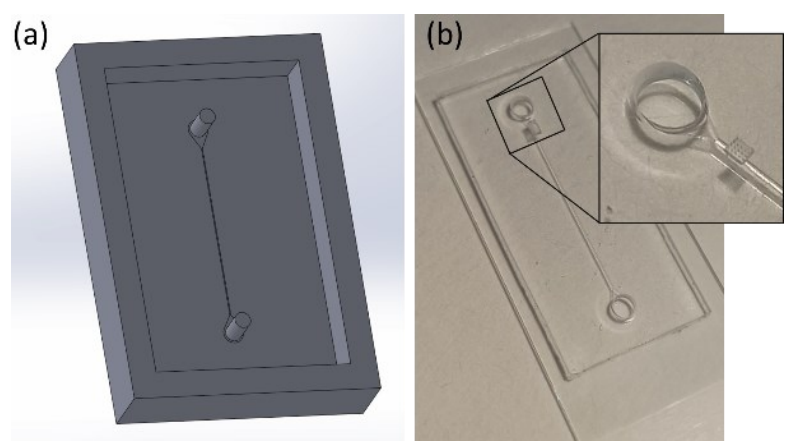

Fig. 4. a) CAD designed master for the microchannel. b) Picture of the final microfluidic device obtained after the bonding of the PDMS sheets to the microscope slide.

\section{Conclusions}

Microfluidics is a multidisciplinary field that requires different technologies capable of manufacturing highly accurate structures in a reliable and precise way. 3D printing has the potential to revolutionize these areas, simplifying manufacturing processes through simple procedures and very low waste production. For its part, laser ablation proves to be a versatile and much more userfriendly technique than photolithography to perform detailed surface micropatterning (unreachable for many current 3D printers).

In this work, a hybrid technique that combines both technologies is proposed, taking advantage of each of them in the range of dimensions in which they show most suitable results. For this, structures of hundreds of microns were printed using a SLA 3D printer. This structures, manufactured in printing resin, were later selective micropatterned by direct laser ablation to obtain inverted microlenses of tens of microns on its surface. Finally, these patterns can be replicated using transparent polymers (as PDMS) to obtain arrays of functional microlenses. This technique allows to manufacture accurate devices such as the one proposed: a microchannel (obtained from a 3D printed master) coupled with an array of microlenses (obtained from a 3D printed master micropatterned by PLA).
Acknowledgements: This work has been sponsored by contracts AEI RTI2018-097063-B-100, AEI/FEDER, UE; ED431B 2020/29; ED431E 2018/08 and ED481D2021-019, Consellería de Educación Xunta de Galicia/FEDER e Estructuración Xunta de Galicia, IN607A2019-02.

\section{References}

1. R. H. Liu, M. A. Stremler, K. V. Sharp, M. G. Olsen, J. G Santiago, R. J. Adrian, H. Aref, D. J. Beebe, Journal of microelectromechanical systems, 9.2, 190197 (2000)

2. B. Heidt, R. Rogosic, S. Bonni, J. Passariello-Jansen, D. Dimech, J. W. Lowdon, R. Arreguin-Campos, E. Steen Redeker, K. Eersels, H. Diliën, B. van Grinsven, T. J. Cleij, Phys. Status Solidi. 217.13, 1900935 (2020)

3. K. Piironen, M. Haapala, V. Talman, P. Järvinen, T. Sikanen, Lab Chip, 20.13, 2372-2382 (2020)

4. A. J. Lopez, J. Lamas, J. S. Pozo-Antonio, T. Rivas, A. Ramil, Opt Lasers Eng, 126, 105897 (2020)

5. J. C. McDonald, D. C. Duffy, J. R. Anderson, D. T. Chiu, H. Wu, O. J. A. Schueller, G. M. Whitesides, Electrophoresis, 21.1, 27-40 (2000)

6. T. Nisisako, H. Suzuki, T. Hatsuazawa, Micromachines, 6.10, 1435-1444 (2015). 